\title{
On a decomposition of Thom-Boardman singularities of order two
}

\author{
By \\ Yoshifumi ANDo \\ Dedicated to Professor A. Komatu on his 70th birthday \\ (Communicated by Prof. H. Toda, July 4, 1977)
}

\section{§0. Introduction}

In this paper we consider the space of differentiable maps having no ThomBoardman singularities of certain type and a decomposition of Thom-Boardman singularities.

Let $\Omega$ be an open subbundle of a jet bundle $J^{r}(N, P)$ over $N$. A differentiable map $f: N \rightarrow P$ is called $\Omega$-regular, if the image of $j^{r} f: N \rightarrow J^{r}(N, P)$ is contained in $\Omega$. Let $C_{\Omega}^{\infty}(N, P)$ be the space of $\Omega$-regular differentiable maps with $C^{r}$-topology and $\Gamma_{N}(\Omega)$ the space of differentiable cross-sections of the subbundle $\Omega$ over $N$ with compact-open topology. If $j^{r}: C_{\Omega}^{\infty}(N, P) \rightarrow \Gamma_{N}(\Omega)$ is a weak homotopy equivalence, then $\Omega$ is called integrable.

Let $I$ be an arbitrary series of $r$ non-negative integers. We shall denote by $\Sigma^{I}$ the Thom-Boardman singularities of the jet bundle $\operatorname{Jr}(N, P)$ with symbol $I$. Let $\Omega^{I}$ be the union of all Thom-Boardman singularities $\Sigma^{K}$ with symbol $K$ which is smaller than or equal to $I$ under the lexiographic order.

We denote $n=\operatorname{dim} N$ and $p=\operatorname{dim} P$. In [4], A. du Plessis has shown the following theorem.

Theorem ([4]). Let $I=\left(i_{1}, \ldots, i_{r}\right)$ and let $d^{I}$ be the number of $j$ 's such that $i_{j}-i_{j+1}>1$. Then the inequality $i_{r}>n-p-d^{I}$ implies that $\Omega^{I}$ is integrable.

The purpose of the present paper is to show how the subbundle $\Omega^{I}$ fails to be integrable and how the map $j^{r}: C_{\Omega^{r}}^{\infty}(N, P) \rightarrow \Gamma_{N}\left(\Omega^{I}\right)$ is close to a weak homotopy equivalence when $i_{r} \leq n-p-d^{I}$.

Theorem. Let $r=2$ and $I=(i, j)$. If $p-n+i=2$, then there exists the following decomposition $\left\{\Sigma^{I}(t, c): t\right.$ and $c$ are non-negative integers $\}$ of the ThomBoardman singularities $\Sigma^{I}$ of $J^{2}(N, P)$ :

i) $\Sigma^{I}(t, c)$ is a regular submanifold of $J^{2}(N, P)$,

ii) $\Sigma^{I}(t, c)$ is non-empty if and only if

a) $\Sigma^{I}$ is non-empty

b) either $c=t=i-j$ 


$$
\text { or } c<t<i-j, t \leq 2 c \text { and } i-j-c \leq 2(t-c),
$$

iii) the codimension of $\Sigma^{I}(t, c)$ in $J^{2}(N, P)$ is

$$
(i-j-c)(i-j+2 c-2 t+1)-(t-c)(2 c-t)+2 i+j(i+1),
$$

iv) $\Sigma^{I}$ is the union of all $\Sigma^{I}(t, c)$,

v) $\Omega^{I}-\bigcup_{\substack{h \leq j \\ j-i+t<0 \\ c \leq t}} \sum^{i, h}(t, c)$ is integrable.

Corollary. Let $I=(i, 0)$ and $p-n+i \geq 2$. Then the induced homomorphism $\left(j^{2}\right)_{*}: \pi_{k}\left(C_{\Omega^{I}}^{\infty}(N, P)\right) \rightarrow \pi_{k}\left(\Gamma_{N}\left(\Omega^{I}\right)\right)$ is isomorphic for $k<3 i-n$ and surjective for $k=3 i-n$.

For general $r$ we shall obtain Theorem 4.1 which is a direct consequence of Transversality lemma due to A. du Plessis [5] and his Theorem ([4]). It does not follow from Theorem 4.1 that $\left(j^{2}\right)_{*}$ is surjective for $k=3 i-n$ in the above corollary.

Let $\widetilde{\Omega^{I}}$ be the union of all Thom-Boardman singularities of $\operatorname{Jr}^{r}(N \times \mathbf{R}, P)$. Let $i: J^{r}(N \times \mathbf{R}, P) \rightarrow J^{r}(N, P)$ be the canonical map induced from the inclusion of $N$ into $N \times 0$. Then we know that $i\left(\widetilde{\Omega^{I}}\right)$ is integrable and contained in $\Omega^{I}$.

The decomposition of the singularities $\Sigma^{I}$ into the submanifolds $\Sigma^{K}(t, c)$ is constructed in $\S 1$ and $\S 2$. We show in $\S 3$ that $i\left(\widetilde{\Omega^{I}}\right)$ is represented as the disjoint union of certain submanifolds $\Sigma^{K}(t, c)$. By the transversality argument we obtain the above corollary.

Throughout the paper all manifolds and differentiable maps should be of class $C^{\infty}$ and all manifolds should satisfy the second axiom of countability.

The author would like to thank Professor M. Adachi for his kind advices.

\section{§1. Preliminaries and lemmas}

We begin by recalling the definition of Thom-Boardman singularities of $J^{2}(n, p)$ (see, for example, [7]). Let $\mathbf{R}$ be the field of real numbers. Let $\mathbf{R}\left[x_{1}, \ldots, x_{n}\right]$ be the polynomial ring over $\mathbf{R}$ with $n$ variables $x_{1}, \ldots, x_{n}$ and $\mathfrak{M}$, its unique maximal ideal $\left(x_{1}, \ldots, x_{n}\right)$.

Let $\mathfrak{I}$ be an ideal of $\mathfrak{M} / \mathfrak{M}^{3}$. The Jacobian extension of $\mathfrak{I}$ is defined as follows. Let $f_{1}, \ldots, f_{a}$ be a set of generators of $\mathfrak{I}$. Let $\mathfrak{I}^{\prime}$ denote the ideal generated by all $s \times s$ minor determinants of the Jacobian matrix $\left(\partial f_{i} / \partial x_{j}\right)$. Then we define $\Delta^{s} \mathfrak{I}$ $=\mathfrak{I}+\mathfrak{I}^{\prime}$. We call a set $y_{1}, \ldots, y_{n}$ of $\mathfrak{M} / \mathfrak{M}^{3}$ a system of coordinates, if $y_{1}, \ldots, y_{n}$ span $\mathfrak{M} / \mathfrak{M}^{3}$. If $y_{1}, \ldots, y_{n}$ is a system of coordinates for $\mathfrak{M} / \mathfrak{M}^{3}$, then $\Delta^{s} \mathfrak{I}$ is generated by $f_{1}, \ldots, f_{a}$ and all $s \times s$ minor determinants of Jacobian matrix $\left(\partial f_{i} / \partial y_{j}\right)$.

The rank of a proper ideal $\mathfrak{I}$ denoted by rk $\mathfrak{I}$, is defined as the dimension of $\mathfrak{M}^{2}+\mathfrak{I} / \mathfrak{M}^{2}$. When rk $\mathfrak{I}$ is $s$, we write $\Delta^{s+1} \mathfrak{I}$ by $\delta \mathfrak{I}$.

Let $z_{1}, \ldots, z_{p}$ be a system of coordinates of $\mathbf{R}^{p}$. Let $z \in J^{2}(n, p)$ and $f: \mathbf{R}^{n} \rightarrow \mathbf{R}^{p}$ be any representative of $z$. Then we write $f_{i}=z_{i} \circ f(i=1, \ldots, p)$. We define $\mathfrak{I}(z)$ to be the ideal generated by the images in $\mathfrak{M} / \mathfrak{M M}^{3}$ of $f_{1}, \ldots, f_{p}$. Clearly the ideal $\mathfrak{I}(z)$ depends only on $z$. 
Definition 1.1. By the Boardman symbol of $\mathfrak{I}(z)$ we mean the pair of integers $(i, j)$, where $i=n-\operatorname{rk} \mathfrak{I}(z)$ and $j=n-\operatorname{rk} \delta \mathfrak{I}(z)$.

Definition 1.2. Let $(i, j)$ be a pair of integers. Let $\Sigma^{i, j}$ denote the set $z \in J^{2}(n, p)$ whose Boardman symbol is $(i, j)$. We call the set $\Sigma^{i, j}$ Thom-Boardman singularity of order two

Theorem 1.3. (Boardman [1]) i) The set $\Sigma^{i, j}$ is nonempty if and only if

a) $n \geqq i \geqq j$,

b) $i \geqq n-p$,

and c) if $i=n-p$, then $i=j$.

ii) $\Sigma^{i, j}$ is locally closed in Zariski topology and nonsingular.

iii) The codimension of $\Sigma^{i, j}$ in $J^{2}(n, p)$ is

$$
(p-n+i)\left\{\frac{1}{2} i(i+1)+i-\frac{1}{2}(i-j)(i-j+1)\right\}-j(i-j) .
$$

Now we define the modified $a$-Jacobian extension of an ideal $\mathfrak{I}(z)$ associated with an element $z$ of $J^{2}(n, p)$. Let $f=\left(f_{1}, \ldots, f_{p}\right)$ be any representative of $z$. Let $a$ be $\left(a_{1}, \ldots, a_{p}\right)$ of $\mathbf{R}^{p}$. Consider the $(p, n+1)$ matrix,

$$
D(f, x, a)=\left(\begin{array}{ccc}
\partial f_{1} / \partial x_{1}, \ldots, \partial f_{1} / \partial x_{n} & a_{1} \\
\vdots & \vdots & \vdots \\
\partial f_{p} / \partial x_{1}, \ldots, \partial f_{p} / \partial x_{n} & a_{p}
\end{array}\right)
$$

Definition 1.4. We define the a-Jacobian extension $\Delta_{a}^{s} \mathfrak{I}(z)$ of $\mathfrak{I}(z)$ to be the ideal generated by $\mathfrak{I}(z)$ and all $s \times s$ determinants of the matrix $D(f, x, a)$. When $i=n-\mathrm{rk} \mathfrak{I}(z)$, we put $\delta_{a} \mathfrak{I}(z)=\Delta_{a}^{n-i+2} \mathfrak{I}(z)$.

Clearly $\Delta_{a}^{s} \mathfrak{I}(z)$ does not depend on the representative $f$ of $z$ and a system of coordinates of $\mathbf{R}^{p}$. By the following lemma $\Delta_{a}^{s} \mathfrak{I}(z)$ does not depend on the choice of a system of coordinates for $\mathfrak{M} / \mathfrak{M l}^{3}$.

Lemma 1.5. Let $y_{1}, \ldots, y_{n}$ be a system of coordinates for $\mathfrak{M} / \mathfrak{M}^{3}$. Let $f$ be a representative of $z$ and $f=\left(f_{1}, \ldots, f_{p}\right)$. Then the a-Jacobian extension $\Delta_{a}^{s} \mathfrak{J}(z)$ is generated by $f_{1}, \ldots, f_{p}$ and all $s \times s$ minor determinants of the Jacobian matrix $D(f, y, a)$.

Proof. Since each of systems of coordinates $\left(x_{1}, \ldots, x_{n}\right)$ and $\left(y_{1}, \ldots, y_{n}\right)$ spans $\mathfrak{M}$, we can write $x_{i}=x_{i}(y), y_{i}=y_{i}(x)$ modulo $\mathfrak{M}^{3}$. Hence we have

$$
D(f, x, a)=\left(\left.D(f, y, a)\right|_{y_{i}=y_{i}(x)}\right)\left(\begin{array}{cccc}
\partial y_{1} / \partial x_{1}, \ldots, \partial y_{1} / \partial x_{n} & 0 \\
\vdots & \vdots & \vdots \\
\partial y_{n} / \partial x_{1}, \ldots, \partial y_{n} / \partial x_{n} & 0 \\
0 & , \ldots, & 0 & 1
\end{array}\right)
$$


By the linear algebra it is well known that if $A, B$ are $(s, n+1)$ matrix and $(n+1, s)$ matrix respectively, then $\operatorname{det} A B=\Sigma \operatorname{det} A^{\prime} \operatorname{det} B^{\prime}$ where $A^{\prime}$ and $B^{\prime}$ run over all $(s, s)$ minor matrices of $A$ and $B$. Since the matrix $(\partial y / \partial x)$ is nonsingular, the ideal generated by all $s \times s$ minor determinants of the matrices $D(f, x, a)$ is the same as that of $D(f, y, a)$ at $y_{i}=y_{i}(x)$.

Q.E.D.

Let $L^{r}(m)$ be the group of invertible elements of $r$-jet space $J^{r}(m, m)$. Then we have a canonical action of $L^{r}(n) \times L^{r}(p)$ on $J^{r}(n, p), \mu: L^{r}(n) \times L^{r}(p) \times J^{r}(n, p) \rightarrow$ $J^{r}(n, p)$ defined by $\mu\left(\left(h, h^{\prime}, z\right)\right)=h^{\prime} \circ z \circ h$ where $h \in L^{r}(n), h^{\prime} \in L^{r}(p)$ and $z \in J^{r}(n, p)$.

An element $h \in L^{2}(n)$ induces a ring-isomorphism $h^{*}: \mathfrak{M} / \mathfrak{M l}^{3} \rightarrow \mathfrak{M} / \mathfrak{M}^{3}$ by considering a composition of a polynomial and $h$. The above lemma can be restated in other words as follows.

Lemma 1.6. If $h \in L^{2}(n)$, then we have $\delta_{a} \Im(z \circ h)=\left(h^{*}\right) \delta_{a} \Im(z)$.

Proof. Let $h(x)=\left(h_{1}, \ldots, h_{n}(x)\right)$ and $f(x)=\left(f_{1}, \ldots, f_{p}(x)\right)$. If we put $y_{i}(x)$ $=h_{i}(x)$, then

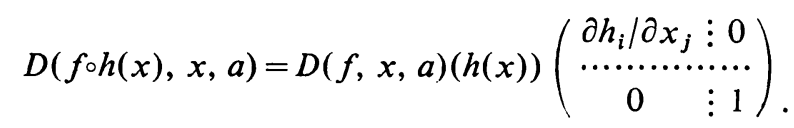

The rest of the proof is similar to that of Lemma 1.5.

Q.E.D.

Lemma 1.7. Let $h \in L^{2}(p)$. Then the ideal $\delta_{a} \mathfrak{I}(z)$ is equal to the ideal $\delta_{b} \mathfrak{I}(h \circ z)$ modulo $\mathfrak{M}^{2}$ where $b$ is defined by

$$
\left(\begin{array}{c}
a_{1} \\
\vdots \\
a_{p}
\end{array}\right)=\left(\begin{array}{cc}
\partial h_{1} / \partial z_{1}(0), \ldots, \partial h_{1} / \partial z_{p}(0) \\
\vdots & \vdots \\
\partial h_{p} / \partial z_{1}(0), \ldots, \partial h_{p} / \partial z_{p}(0)
\end{array}\right)\left(\begin{array}{c}
b_{1} \\
\vdots \\
b_{p}
\end{array}\right) .
$$

Proof. Let $b_{1}(x), \ldots, b_{p}(x)$ be defined by

$$
a_{j}=\sum_{k} \partial h_{j} / \partial z_{k}(z(x)) b_{k}(x) \quad(j=1, \ldots, p) .
$$

Then we have $D(h \circ z, x, a)=\left(\partial h_{j} / \partial z_{k}(z(x)) D(z, x, b(x))\right.$. Let $s=\mathrm{rk} \mathfrak{I}(z)+2$. Since the matrix $\left(\partial h_{j} / \partial z_{k}\right)$ is nonsingular, the ideal generated by all $s \times s$ determinants of $D(h \circ z, x, a)$ is equal to that of $D(z, x, b(x))$ by the similar arguments in the proof of Lemma 1.5. Let $b_{i}=b_{i}(0)(i=1, \ldots, p)$. Then the last ideal is equal to the ideal constructed similarly from $D(z, x, b)$ modulo $\mathfrak{M}^{2}$.

Q.E.D.

Remark 1.8. Let $z$ be an element of $J^{2}(n, p)$. Then the ideal $\delta_{a} \Im(z)$ is contained in the ideal $\delta \mathfrak{I}(z)$ for any $a \in \mathbf{R}^{p}$.

\section{§2. A decomposition of $\boldsymbol{\Sigma}^{\boldsymbol{i}, \boldsymbol{j}}$ into manifolds}

We will give a decomposition of $\Sigma^{i, j}$ into a finite set of nonsingular submanifolds in $J^{2}(n, p)$ which are locally closed in Zariski topology. 
Definition 2.1. By the symbol for decomposition for an element $z$ of $\Sigma^{i, j}$ we mean a pair of integers $(t, c)$ defined as follows. Let $t$ be an integer such that $\operatorname{rk} \delta_{a} \mathfrak{I}(z) \leqq n-i+t$ for any element $a \in \mathbf{R}^{p}$ and that for some element $b \operatorname{rk} \delta_{b} \mathfrak{I}(z)$ $=n-i+t$. Let $U(z)=\left\{a \in \mathbf{R}^{p} \mid \operatorname{rk} \delta_{a} \mathfrak{I}(z)=n-i+t\right\}$. Then the integer $c$ is defined to be the rank of the ideal $\underset{a \in U(z)}{\cap} \delta_{a} \mathfrak{I}(z)$.

Remark 2.2. For an element $z$ of the singularity $\Sigma^{i, j}$, it follows from Remark 1.8 that $t \leqq i-j$. It is clear that $c \leqq t$. The set $U(z)$ is a Zariski open set in $\mathbf{R}^{p}$ since the inequality $\mathrm{rk} \delta_{a} \mathfrak{I}(z) \leqq n-i+t$ is an algebraic condition.

Definition 2.3. Let $(t, c)$ be a pair of nonnegative integers. Let $\Sigma^{i, j}(t, c)$ denote the set of those elements $z \in \Sigma^{i, j}$ whose symbol for decomposition is $(t, c)$.

Remark 2.4. $\Sigma^{i, j}(t, c)$ is $L^{2}(n) \times L^{2}(p)$ invariant; In fact it is known that $\Sigma^{i, j}$ is $L^{2}(n) \times L^{2}(p)$ invariant. Let $h \in L^{2}(n)$. Then it follows from Lemma 1.6 that the symbols for decomposition of $z$ and $z$ oh are equal. Hence $\Sigma^{i, j}(t, c)$ is $L^{2}(n)$ invariant. Let $h^{\prime} \in L^{2}(p)$. By Lemma 1.7 we have $\bigcap_{U(z) \ni a} \delta_{a} \Im(z)=\bigcap_{U\left(h^{\prime} \circ z\right) \ni b}$ $\delta_{h} \mathfrak{J}\left(h^{\prime} \circ z\right)$. Hence $\Sigma^{i, j}(t, c)$ is also $L^{2}(p)$ invariant.

Let $n-i \leqq n-i+c \leqq n-j$. Let $G L_{n-i, n-i+c, n-j, j}\left(\mathfrak{M M} / \mathfrak{M}^{2}\right)$ be the set of the following series of three vector subspaces of $\mathfrak{M} / \mathfrak{M}^{2},\{(\alpha, \beta, \gamma) \mid \alpha, \beta, \gamma$ are subspaces of $\mathfrak{M} / \mathfrak{M}^{2}, \operatorname{dim} \alpha=n-i, \operatorname{dim} \beta=n-i+c$ and $\left.\operatorname{dim} \gamma=n-j . \quad \alpha \subseteq \beta \cong \gamma\right\} . \quad$ We denote this grassmannian manifold by $G L_{*}$ for simplicity. Let $G L_{n-i, p-n+i}\left(\mathbf{R}^{p}\right)$ be the grassmannian manifold of subspaces of dimension $n-i$ in $\mathbf{R}^{p}$.

We will define a map $\varphi: \Sigma^{i, j}(t, c) \rightarrow G L_{*} \times G L_{n-i, p-n+i}\left(\mathbf{R}^{p}\right)$. Let $z$ be an element of $\Sigma^{i, j}(t, c)$. Then we obtain a triple of vector subspaces $(\alpha, \beta, \gamma)$ where $\alpha=\mathfrak{I}(z), \beta=\underset{U(z) \ni a}{\bigcap} \delta_{a} \mathfrak{I}(z)$, and $\gamma=\delta \mathfrak{I}(z)$ modulo $\mathfrak{M}^{2}$. By definition of $\Sigma^{i, j}(t, c)$, we have $\operatorname{dim} \alpha=n-i, \operatorname{dim} \beta=n-i+c$ and $\operatorname{dim} \gamma=n-j$ with $\alpha \subseteq \beta \leqq \gamma$. Hence $(\alpha, \beta, \gamma) \in G L_{*}$. Consider the differential map at the origin of $\mathbf{R}^{n}, \mathrm{~d} f_{0}: T_{0} \mathbf{R}^{n} \rightarrow$ $T_{0} \mathbf{R}^{p}$. Then we put $=\operatorname{Imd} f_{0}\left(T_{0} \mathbf{R}^{n}\right)$ which is in $G L_{n-i, p-n+i}$.

Lemma 2.5. $\left(\Sigma^{i, j}(t, c), \varphi, G L^{*} \times G L_{n-i, p-n+i}\left(\mathbf{R}^{p}\right)\right)$ is a fiber bundle.

Proof. In Remark 2.4 we have defined the action of $L^{2}(n) \times L^{2}(p)$ on $\Sigma^{i, j}(t, c)$. We restrict this action to $L^{1}(n) \times L^{1}(p)$. Then it follows from Lemma 1.6 and Lemma 1.7 that the following diagram commutes.

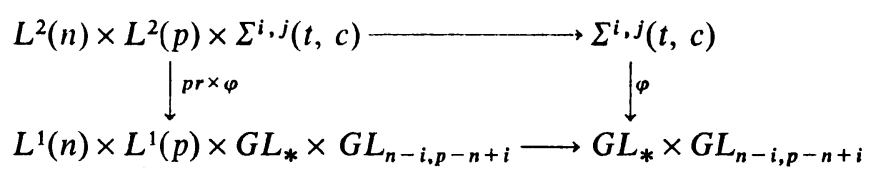

Since $L^{1}(n)$ and $L^{1}(p)$ act transitively on $G L_{*}$ and $G L_{n-i, p-n+i}$ respectively, $G L_{*}$ $\times G L_{n-i, p-n+i}$ is a homogeneous space of $L^{1}(n) \times L^{1}(p)$. Hence there is a local cross section of $G L_{*} \times G L_{n-i, p-n+i}$ into $L^{1}(n) \times L^{1}(p)$. It follows from Lemma 1 of Cerf $[2$, Appendix $]$ that $\varphi$ is a fiber bundle.

Q.E.D.

The next purpose is to show that $\varphi$ gives a differentiable fiber bundle. At 
first we will show that the fibre of the bundle $\varphi$ is a differentiable manifold.

We fix $((\alpha, \beta, \gamma), \delta)$ for a while. Let $\left(x_{1}, \ldots, x_{n}\right)$ be a system of coordinates such that $\alpha, \beta$ and $\gamma$ are spanned by the images of $\left(x_{1}, \ldots, x_{n-i}\right),\left(x_{1}, \ldots, x_{n-i+c}\right)$ and $\left(x_{1}, \ldots\right.$, $\left.x_{n-j}\right)$ by the natural map into $\mathfrak{M} / \mathfrak{M}^{2}$, respectively. Let $y_{1}, \ldots, y_{p}$ be a system of coordinates such that $\operatorname{Imd} f_{0}$ of the tangent space $T_{0}\left(\mathbf{R}^{n}\right)$ is spanned by $\partial / \partial y_{1}, \ldots$, $\partial / \partial y_{n-i}$. Under these systems of coordinates we can identify an element of $z \in J^{2}(n$, $p)$ with a polynomial of order $2\left(b_{s t}=b_{t s}\right)$,

$$
f^{k}=\Sigma a_{j}^{k} x_{j}+\Sigma b_{i j}^{k} x_{i} x_{j} \quad(k=1, \ldots, p) .
$$

We now search for the explicit conditions for $z$ so that $z$ is contained in $\varphi^{-1}((\alpha$, $\beta, \gamma), \delta)$. Let $z$ be in $\varphi^{-1}((\alpha, \beta, \gamma), \delta)$. Then it is easy to see that $a_{t}^{s}=0$ when $s>n-i$ or $t>n-i$, since $\mathfrak{I}(z)=\left(x_{1}, \ldots, x_{n-i}\right)$ modulo $\mathfrak{M}^{2}$ and the image of $\mathrm{d} f: T_{0} \mathbf{R}^{n} \rightarrow T_{0} \mathbf{R}^{n}$ is spanned by $\partial / \partial y_{1}, \ldots, \partial / \partial y_{n-i}$. The $(n-i, n-i)$ minor matrix $\left(a_{t}^{s}\right)_{1 \leqq s, t \leqq n-i}$ is nonsingular.

Under (2.6), $\delta_{a} \mathfrak{I}(z)$ is described more explicitly. We note that $D(f, x, a)$ is the following matrix;

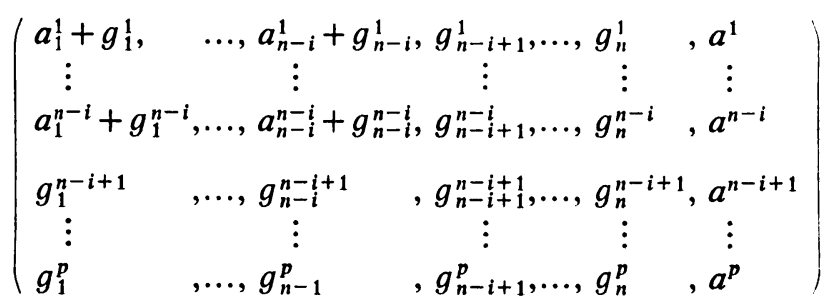

where $g_{t}^{s}=\partial\left(\Sigma b_{i j}^{s} x_{i} x_{j}\right) / \partial x_{t}=\sum_{j=1}^{n}\left(b_{t j}^{s}+b_{j t}^{s}\right) x_{j}$. By definition of $\delta_{a} \mathfrak{I}(z)$, it is an ideal generated by $\mathfrak{I}(z)$ and all $(n-i+2, n-i+2)$ determinants of $D(f, x, a)$. It follows from the form of matrix $\left(a_{t}^{s}\right)$ that the image of $\delta_{a} \mathfrak{I}(z)$ into $\mathfrak{M} / \mathfrak{M}^{2}$ is generated by $\sum_{t=1}^{n-1} a_{t}^{s} x_{t}(1 \leqq s \leqq n-i)$ and $a^{s} g_{u}^{t}-a^{t} g_{u}^{s}(n-i+1 \leqq s, t \leqq p$ and $n-i+1 \leqq u \leqq p)$.

The module $\mathfrak{M} / \mathfrak{M}^{2}$ is isomorphic to the vector space $\mathbf{R}^{n}$ by mapping $\Sigma \lambda_{i} x_{i}$ into the row vector $\left(\lambda_{1}, \lambda_{2}, \ldots, \lambda_{n}\right)$. Let $e_{i}$ correspond to $x_{i}(i=1, \ldots, n)$. Let $A$ be the matrix $\left(a_{t}^{s}\right)_{1 \leqq s \leqq p, 1 \leqq t \leqq n}, \bar{B}^{j},\left(b_{s t}^{j}\right)_{1 \leqq s, t \leqq n}(j=1, \ldots, p)$ and $B^{j}$ the $(i, i)$ minor matrix of $\bar{B}^{j},\left(b_{s t}^{j}\right)_{n-i+1 \leqq s, \imath \leqq n}$. By the notation $V(X)$ we shall mean the vector subspace spanned by all row vectors of a matrix $X$. The image of $\delta_{a} \mathfrak{I}(z)$ onto $\mathfrak{M} / \mathfrak{M}^{2}$ is identified with the sum of vector subspaces, $V(A)$ and $V\left(a^{l} \bar{B}^{m}-a^{m} \bar{B}^{l}\right), n-i+1 \leqq l, m \leqq p$ of $\mathbf{R}^{n}$. It follows from the form of $A$ that the above space is generated by $e_{1}, \ldots, e_{n-i}$ and $V\left(a^{l} B^{m}-a^{m} B^{l}\right), n-i+1 \leqq l, m \leqq p$. We note that $\operatorname{rk} \delta_{a} \Im(z)=n-i+t$ if and only if the dimension of the subspace spanned by $V\left(a^{l} B^{m}-a^{m} B^{l}\right), n-i+1 \leqq l$, $m \leqq p$ (which will be denoted for convenience by $V(B, a)$ hereafter) is $t$. Let $U(B)=\left\{a \in \mathbf{R}^{p-n+i} \mid \operatorname{dim} V(B, a)=t\right\}$. Similarly $\delta \mathfrak{I}(z)$ is identified with the subspace generated by $e_{1}, \ldots, e_{n-j}$ and $V\left(B^{j}\right), n-i+1 \leqq j \leqq p$ of $\mathbf{R}^{n}$. Hence we have the following lemma.

Lemma 2.7. Let $z$ be an element of $\Sigma^{i, j}(t, c)$ and represented as in 2.6. 
Then $\mathfrak{I}(z)=\left(x_{1}, \ldots, x_{n-i}\right), \delta \mathfrak{I}(z)=\left(x_{1}, \ldots, x_{n-j}\right)$ and $\underset{U(z) \ni a}{\cap} \delta_{a} \mathfrak{I}(z)=\left(x_{1}, \ldots, x_{n-i+c}\right)$ modulo $\mathfrak{M}^{2}$ if and only if

i) the $(n-i, n-i)$ minor matrix $\left(a_{t}^{s}\right)_{1 \leqq s, t \leqq n-i}$ is nonsingular and $a_{t}^{s}=0$ if $s$ or $t>n-i$,

ii) $\sum_{j=n-i+1}^{p} V\left(B^{j}\right)=\left\{e_{n-i+1}, \ldots, e_{n-j}\right\}$,

iii) $\operatorname{dim} V(B, a) \leqq t$ and $U(B) \neq \varnothing$,

iv) $\underset{U(B) \ni a}{ } V(B, a)=\left\{e_{n-i+1}, \ldots, e_{n-i+c}\right\}$.

In the rest of this section we assume $p-n+i=2$. We note that $J^{2}(n, p)$ is identified with the product of the space of $(n, p)$ matrices and $p$ powers of the space $A_{n}$ of $(n, n)$ symmetric matrices. Now we give a proof of Theorem except for $\left.\mathrm{v}\right)$.

Proof of Theorem. The fibre $\varphi^{-1}((\alpha, \beta, \gamma), \delta)$, which is denoted by $F$ here after, is identified with the space of those elements of $J^{2}(n, p)$ whose representations as in (2.6) satisfy the conditions i), ii), iii) and iv) of Lemma 2.7. Since $p-n+i=2$, $F$ is identified with the product of the space of nonsingular $(n-i, n-i)$ matrices, $\left(A_{n}\right)^{n-i}$ and $\tilde{\Sigma}$ where $\widetilde{\Sigma}$ denotes the following subspace of $A_{n} \times A_{n}$. Let $\bar{B}=\left(b_{i j}\right)$ be $n$-th symmetric matrices and $B$-th symmetric matrices $\left(b_{i j}\right)_{n-i+1 \leqq i, j \leqq n}$. We define a map $\pi: A_{n} \rightarrow A_{i}$ by $\pi(\bar{B})=B$. We let $\Sigma^{\prime}$ be the subspace of all $\left(B^{1}, B^{2}\right)$ of $\left(A_{i}\right)^{2}$ such that the conditions ii), iii) and iv) holds for $\left(B^{1}, B^{2}\right)$. Then $\tilde{\Sigma}$ is $\pi^{-1}\left(\Sigma^{\prime}\right)$. Therefore it follows from Lemma 2.11 that $F$ is nonsingular in $J^{2}(n, p)$. It is easy to see that $\operatorname{dim} F=(n-i)^{2}+\frac{1}{2} n(n-i)(n+1)+2\left\{i(n-i)+\frac{1}{2}(n-i)(n-i+1)\right\}+(t-c)$ $(2 c-t)+2\left\{\frac{1}{2} c(c+1)+(t-c)(i-j-c)\right\}$.

We consider the differentiable map $\mu: L^{1}(n) \times L^{1}(p) \times F \rightarrow J^{2}(n, p)$ defined by $\mu\left(h, h^{\prime}\right)(z)=h^{\prime} \circ z \circ h$. At first we show that the image of $\mu$ is $\Sigma^{i, j}(t, c)$. It is clear that the image of $\mu$ is contained in $\Sigma^{i, j}(t, c)$ since $F$ is a subset of $\Sigma^{i, j}(t, c)$ (Remark 2.4). Consider the following diagram,

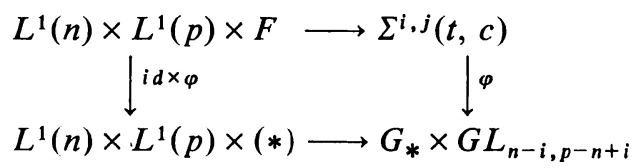

where $(*)$ means $((\alpha, \beta, \gamma), \delta)$. Since $L^{1}(n) \times L^{1}(p)$ acts on $G^{*} \times G L_{n-i, p-n+i}$ transitively, the image of $\mu$ is $\Sigma^{i, j}(t, c)$. Next we show that $\mu$ is of constant rank. It is enough to show that $\mu$ is of constant rank at the unit element 1 of $L^{1}(n) \times L^{1}(p)$. We put $\mu(v, z)=\mu_{v}(z)=\mu_{z}(v)$. Let $(\mathrm{d} \mu)_{1, z}(x, y)=0$ where $x \in T_{1}\left(L^{1}(n) \times L^{1}(p)\right)$ and $y \in T_{z} F$. Since $(\mathrm{d} \mu)_{1, z}=\mathrm{d}\left(\mu_{z}\right)_{1}+\mathrm{d}\left(\mu_{x}\right)_{z}$, we have $\mathrm{d}\left(\mu_{z}\right)_{1}(x)+\mathrm{d}\left(\mu_{1}\right)_{z}(y)=0$. As $\mu_{1}: F \rightarrow J^{2}(n, p)$ is an embedding, $\mathrm{d}\left(\mu_{z}\right)_{1}(x)$ determines $y$. Since $\mathrm{d} \varphi \circ \mathrm{d}\left(\mu_{1}\right)_{z}(y)$ $=\mathrm{d}\left(\varphi \circ \mu_{1}\right)_{z}(y)=0, x$ is in $\operatorname{Ker} \mathrm{d} \varphi \circ \mathrm{d}\left(\mu_{z}\right)_{1}$. Hence $\operatorname{dim} \operatorname{Ker} \mathrm{d}\left(\varphi \circ \mu_{z}\right)_{1}$ is equal to $\operatorname{dim} \operatorname{Ker}(\mathrm{d} \mu)_{1, z}$. Now we have

$$
\begin{aligned}
\mathrm{rk}_{1, z} \mathrm{~d} \mu & =\operatorname{dim} T_{1}\left(L^{1}(n) \times L^{1}(p)\right)+\operatorname{dim} T_{z} F-\operatorname{dim} \operatorname{Ker}(\mathrm{d} \mu)_{1, z} \\
& =\operatorname{dim} T_{1}\left(L^{1}(n) \times L^{1}(p)\right)-\operatorname{dim} \operatorname{Ker} \mathrm{d}\left(\varphi \circ \mu_{z}\right)_{1}+\operatorname{dim} T_{z} F
\end{aligned}
$$




$$
=\operatorname{dim} G_{*} \times G L_{n-i, p-n+i}+\operatorname{dim} F .
$$

Finally we assert that $\mu: L^{1}(n) \times L^{1}(p) \times F \rightarrow \Sigma^{i, j}(t, c)$ is an open map. This follows from Lemma 2.5. Hence by the constant rank theorem ([3, Chapter X, Section 3]), $\Sigma^{i, j}(t, c)$ is nonsingular with $\operatorname{dim} G_{*} \times G L_{n-i, p-n+i}+\operatorname{dim} F$. Moreover since $\mu$ is a proper map, $\Sigma^{i, j}(t, c)$ is a regular submanifold of $J^{2}(n, p)$. Hence $\Sigma^{i, j}(t, c)$ is a regular submanifold of $\Sigma^{i, j}$.

Now we determine the dimension of $\Sigma^{i, j}(t, c)$ in $J^{2}(n, p)$. Consider canonical bundles over grassmannian manifolds, $G L_{n-i, n-i+c, n-j, j}\left(\mathbf{R}^{n}\right) \rightarrow G L_{n-j, j}\left(\mathbf{R}^{n}\right)$ with fibre $G L_{n-i, n-i+c, i-c-j}\left(\mathbf{R}^{n-j}\right)$ and $G L_{n-i, n-i+c, i-c-j}\left(\mathbf{R}^{n-j}\right) \rightarrow G L_{n-i+c, i-c-j}\left(\mathbf{R}^{n-j}\right)$ with fibre $G L_{n-i, c}\left(\mathbf{R}^{n \mid i+c}\right)$. Then we have that $\operatorname{dim} G_{*}=\operatorname{dim} G L_{n-i, c}+\operatorname{dim} G L_{n-i+c, i-c-j}+$ $\operatorname{dim} G L_{n-j, j}=(n-i) c+(n-i+c)(i-c-j)+(n-j) j$. Hence

$$
\begin{aligned}
\operatorname{dim} \Sigma^{i, j}(t, c)= & (n-i)\left\{(n-i)+\frac{1}{2} n(n+1)\right\}+2 i(n-i)+2(t-c) \\
& \times(i-j-c)+(n-i)(n-i+1)+c(c+1)+(t-c)(2 c-t) \\
& +(n-i) c+(n-i+c)(i-c-j)+(n-j) j+2(n-i) .
\end{aligned}
$$

The codimension of $\Sigma^{i, j}(t, c)$ follows from $\operatorname{dim} J^{2}(n, p)=p\left\{n+\frac{1}{2} n(n+1)\right\}$.

Since $J^{2}(N, P)$ is a bundle over $N \times P$ with fibre $J^{2}(N, P)_{x, y}$ over $(x, y)$ which is identified with $J^{2}(n, p)$, we can define $\Sigma^{i, j}(t, c)_{x, y}$ in $J^{2}(N, P)_{x, y}$. Let $\Sigma^{i, j}(t, c)$ $=\bigcup_{x \in N, y \in P} \sum^{i, j}(t, c)_{x, y}$. It follows from Remark 2.4 that $\Sigma^{i, j}(t, c)$ is a submanifold of $J^{x \in N, y \in P}(N, P)$.

Q.E.D.

Remark 2.8. $\Sigma^{i, j}(t, c)$ is locally closed in Zariski topology. For $\Sigma^{i, j}$ is locally closed in Zariski topology. The set $S_{t}$ of all $z$ so that $\operatorname{rk} \delta_{a} \Im(z) \leqq n-i+t$ is closed in Zariski topology. The set $T_{c}$ of all $z$ so that $\mathrm{rk} \underset{U(z) \ni a}{\cap} \delta_{a} \Im(z) \leqq n-i+c$ is also closed. Hence $S_{t}-S_{t-1}$ and $T_{c}-T_{c-1}$ are locally closed in Zariski topology of $\Sigma^{i, j}$. Then $\Sigma^{i, j}(t, c)=\left(S_{t}-S_{t-1}\right) \cap\left(T_{c}-T_{c-1}\right)$. This is what we want.

Lemma 2.9. Let $B^{1}$ and $B^{2}$ be $i \times i$ symmetric matrices. Let $\operatorname{dim} V(B, a)$ $\leqq t, U(B) \neq \varnothing, \bigcap_{U(B) \ni a} V(B, a)=\left\{e_{1}, \ldots, e_{c}\right\}$, and $V\left(B^{1}\right)+V\left(B^{2}\right)=\left\{e_{1}, \ldots, e_{i}\right\}$. Then there exists an element $T$ with the form $\left(\begin{array}{cc}S_{1} & 0 \\ * & S_{2}\end{array}\right)$, where $S_{1} \in G L(c)$ and $S_{2} \in G L(i-c)$ as follows.

i) ${ }^{t} T B^{j} T=\left(\begin{array}{ccc}* & * & X_{1}^{j} \\ * & X_{2}^{j} & 0 \\ t X_{1}^{j} & 0 & 0\end{array}\right)$ where $X_{2}^{j}$ are symmetric $(2 c-t, 2 c-t)$ matrices, $X_{1}^{j}$ are $(i-c, t-c)$ nonsingular matrices and $\operatorname{dim} V\left(X_{2}^{1}\right)+V\left(X_{2}^{2}\right)=2 c-t$.

ii) The intersection of all $V\left(a^{2} X_{1}^{1}-a^{1} X_{1}^{2}\right)$ is $\{0\}$, where $a$ is in $\left\{a \in R^{2}\right.$ $\left.\operatorname{rk}\left(a^{2} X_{1}^{1}-a^{1} X_{1}^{2}\right)=t-c\right\}$.

iii) For some $a \in \mathbf{R}^{2}, \operatorname{rk}\left(a^{2} X_{1}^{1}-a^{1} X_{1}^{2}\right)=t-c$.

iv) $\operatorname{dim} V\left(X_{1}^{1}\right)+V\left(X_{1}^{2}\right)=i-c$. 
Proof. In the proof let $V(X)$ denote the subspace generated by all column vectors of $X$. Since $B^{1}$ and $B^{2}$ are symmetric, it does not matter. At first we only consider $B^{2}$. Let $C$ be the intersection of all vector spaces $V(B, a), a \in U(B)$. Let $d$ be the dimension of $V\left(B^{2}\right)+C / C$. Then there is an element $T_{1} \in G L(i)$ such that $B^{2} T_{1}$ is $\left(b_{1}^{\prime}, \ldots, b_{i}^{\prime}\right)$ where $b_{d+1}^{\prime}, \ldots, b_{i}^{\prime}$ are contained in $V\left(B^{2}\right) \cap C, b_{1}^{\prime}, \ldots, b_{d}^{\prime}$ are not contained in $V\left(B^{2}\right) \cap C$ and linearly independent. Hence if ${ }^{t} T_{1} B^{2} T_{1}$ is written as $\left(b_{1}^{2}, \ldots, b_{i}^{2}\right)$, then $b_{d+1}^{2}, \ldots, b_{i}^{2}$ are contained in $V\left({ }^{t} T_{1} B^{2} T_{1}\right) \cap{ }^{t} T_{1}(C), b_{1}^{2}, \ldots, b_{d}^{2}$ are not and $b_{1}^{2}, \ldots, b_{d}^{2}$ are linearly independent. Here ${ }^{t} T_{1}(C)$ is the intersection of all $V\left({ }^{t} T_{1} B T_{1}, a\right), a \in U(B)$. Let ${ }^{\prime} T_{1} B^{1} T_{1}$ be written as $\left(b_{1}^{1}, \ldots, b_{i}^{1}\right)$. The vector $a^{2} b_{j}^{1}-a^{1} b_{j}^{2}$ is contained in $V\left({ }^{t} T_{1} B T_{1}, a\right)$. If $j>d$ and $a \in U$, then $a^{1} b_{j}^{2}$ is an element of ${ }^{t} T_{1}(C)$. Hence $a^{2} b_{j}^{1}$ is contained in $V\left({ }^{t} T_{1} B T_{1}, a\right)$, and so is $b_{j}^{1}$ if $a^{2} \neq 0$. Let $\left(a^{1}, 0\right) \in U(B)$. Then $\operatorname{dim} V\left({ }^{t} T_{1} B T_{1}, a\right)$ is constantly $t$ for $a \in U(B)$ and there is a sequence of points in $U(B)$ which converges a point $\left(a^{1}, 0\right)$. Therefore, $b_{j}^{1}$ is also an element of $V\left({ }^{t} T_{1} B T_{1}, a\right)$ for $a^{2}=0$. Hence $b_{j}^{1} \in{ }^{t} T_{1}(C)$ if $j>d$.

Similarly we can prove that $b_{1}^{1}, \ldots, b_{d}^{1}$ are not contained in ${ }^{t} T_{1}(C)$ and linearly independent. In fact let $\sum_{i=1}^{d} \lambda_{i} b_{i}^{1}=0$. Then $a^{2}\left(\Sigma \lambda_{i} b_{i}^{2}\right)-a^{1}\left(\Sigma \lambda_{i} b_{i}^{2}\right) \in V\left({ }^{\prime} T_{1} B T_{1}, a\right)$, hence we have $a^{1}\left(\sum \lambda_{i} b_{i}^{2}\right) \in V\left({ }^{t} T_{1} B T_{1}, a\right)$. By the similar argument as above $\Sigma \lambda_{i} b_{i}^{2}$ is contained in ${ }^{\prime} T_{1}(C)$. This means $\lambda_{1}=\cdots=\lambda_{d}=0$.

Let $\pi_{2}$ denote the projection of $\mathbf{R}^{i}$ into $\mathbf{R}^{i-d}$. Let $\mathbf{R}^{d}$ (or $\mathbf{R}^{i-d}$ ) is spanned by $e_{1}, \ldots, e_{d}\left(\right.$ or $\left.e_{d+1}, \ldots, e_{i}\right)$. Let $\operatorname{dim}^{t} T_{1}(C) \cap\left\{e_{1}, \ldots, e_{d}\right\}$ is $d_{1}$ and $\operatorname{dim} \pi_{2}\left({ }^{t} T_{1}(C)\right)$ is $d_{2}\left(d_{1}+d_{2}=c\right)$. There exist $S_{1} \in G L(d), S_{2} \in G L(i-d)$ such that the linear map ${ }^{t} S_{1}: \mathbf{R}^{d} \rightarrow \mathbf{R}^{d}$ (or ${ }^{t} S_{2}: \mathbf{R}^{i-d} \rightarrow \mathbf{R}^{i-d}$ ) sends ${ }^{t} T_{1}(C) \cap \mathbf{R}^{d}$ (or $\pi_{2}\left({ }^{t} T_{1}(C)\right.$ )). onto the subspace $\left\{e_{1}, \ldots, e_{\dot{d}_{1}}\right\}$ (or $\left\{e_{d+1}, \ldots, e_{d+d_{2}}\right\}$ ). If we transform matrices ${ }^{t} T_{1} B^{j} T_{1}$. by $T_{2}=S_{1} \times S_{2}$, then they have the following forms.

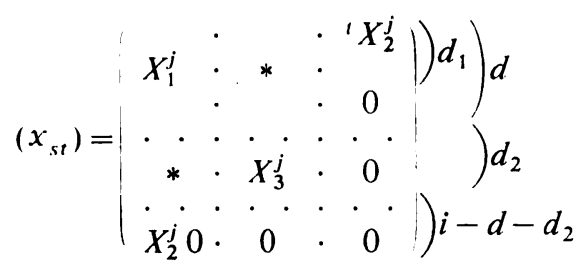

where $x_{s t}=0(s \geqq t)$ if $s>d_{1}$ and $t>d+d_{2}$. For if we write ${ }^{t} T_{1} B^{j} T_{1}=\left(\begin{array}{cc}X_{1} & { }^{t} X_{2} \\ X_{2} & X_{3}\end{array}\right)$ where $X_{1}$ is a $(d, d)$ matrix, $X_{2}$ a $(i-d, d)$ matrix, $X_{3}$ a $(i-d, i-d)$ matrix, then $V\left(X_{3}\right) \in \pi_{2}\left({ }^{t} T(C)\right)$ and $V\left({ }^{t} S_{2} X_{3}\right) \subseteq\left\{e_{d+1}, \ldots, e_{d+d_{2}}\right\}$. We put $T=T_{1} \cdot T_{2}$.

Now we show $d_{1}=d$. Let $x_{h}^{j}$ be the $h$-th column vector of ${ }^{t} T B^{j} T$. Let $W(a)$ be the space generated by $a^{2} x_{d_{1}+1}^{1}-a^{1} x_{d_{1}+1}^{2}, \ldots, a^{2} x_{d}^{1}-a^{1} x_{d}^{2}$ of dimension $d-d_{1}$. It follows from $(*)$ that $W(a)$ is contained in the space $\left\{e_{1}, \ldots, e_{d+d_{2}}\right\}$. By the above construction $W(a) \cap\left({ }^{t} T\right)(C)=0$. Otherwise there exists an element $\sum_{h=d_{1}+1}^{d} \lambda_{h}\left(a^{2} x_{h}^{1}\right.$ $\left.-a^{1} x_{h}^{2}\right)$ of $\left.{ }^{t} T\right)(C)$. Hence $t \sum_{h} \lambda_{h}\left(a^{2} x_{h}^{1}-a^{1} x_{h}^{2}\right)+s a^{1} \sum_{h} \lambda_{h} x_{h}^{2}$ is in $V\left({ }^{t} T B T, b\right)$ where $b_{1}=s a^{1}-t a^{1}$ and $b_{2}=t a^{2}$. Hence $\left(\sum \lambda_{h} x_{h}^{2}\right) \in V\left({ }^{t} T B T, b\right), b \in U(B)$. This is a contradiction. Since $\left({ }^{\prime} T\right)(C)$ is a subspace of $\operatorname{dim} d_{1}+d_{2}$ in $\left\{e_{1}, \ldots, e_{d+d_{2}}\right\}, W(a)$ 
$+\left({ }^{t} T\right)(C)=\left\{e_{1}, \ldots, e_{d+d_{2}}\right\}$ for $a \in U(B)$. This shows that the subspace $\left\{e_{1}, \ldots, e_{d+d_{2}}\right\}$ is contained in $V\left({ }^{t} T B T, a\right)$ for any $a \in U(B)$. That is, $\left\{e_{1}, \ldots, e_{d+d_{2}}\right\}$ is contained in $\left({ }^{t} T\right)(C)$. By compairing dimensions of $\left\{e_{1}, \ldots, e_{d+d_{2}}\right\}$ and $C$, we obtain $d_{1}=d$.

We here note that the intersection of vector spaces $V\left({ }^{t} T B T, a\right), a \in U(B)$ is $\left\{e_{1}, \ldots, e_{c}\right\}$. This means that the intersection $C$ is preserved by the transformation $T$. Hence $T\left(=T_{1} \cdot T_{2}\right)$ has the following form, $\left(\begin{array}{ll}S_{1} & 0\end{array}\right)$, where $S_{1}$ and $S_{2}$ are $c \times c$ $\left(\begin{array}{ll}S_{1} & 0 \\ * & S_{3}\end{array}\right)$

matrix and $(i-c) \times(i-c)$ matrix. Since $c=d_{1}+d_{2}$ and $t=d_{1}+c$, we have $d_{1}=t-c$ and $d_{2}=2 c-t$.

Q.E.D.

Let $\Sigma$ denote the space of all pairs $\left(B^{1}, B^{2}\right)$ of $i$-th symmetric matrices with $\operatorname{dim} V(B, a) \leqq t, U(B) \neq \varnothing, \underset{U(B) \ni a}{\bigcap} V(B, a)=\left\{e_{1}, \ldots, e_{c}\right\}$ and $V\left(B^{1}\right)+V\left(B^{2}\right)=\left\{e_{1}, \ldots, e_{i}\right\}$.

Corollary 2.10. The space $\Sigma$ is nonempty if and only if i) $2 c-t \geqq 0, i>t>c$ and $2(t-c) \geqq i-c$ or ii) $c=t=i-j$.

Proof. Necessity follows from Lemma 2.9. We give an example $\left(B^{1}, B^{2}\right)$ with the conditions of the lemma. Under the notations of Lemma 2.9, let ${ }^{t} X_{1}$ $=\left(\begin{array}{c}E_{t-c} \\ 0\end{array}\right)$ and ${ }^{t} X_{1}^{2}=\left(\begin{array}{cc}0 & E_{2 t-c-i} \\ 0 & 0 \\ E_{i-t} & 0\end{array}\right.$

Q.E.D.

We now define a map $\varphi: \Sigma \rightarrow G L_{t-c, 2 c-t}\left(\mathbf{R}^{c}\right)$. Let $\varphi\left(\left(B^{1}, B^{2}\right)\right)$ be the space of the intersection of $V\left(a^{1} B^{2}-a^{2} B^{1}\right), a \in R^{2}-\{0\}$. It is easily seen from Lemma 2.9 that $\varphi\left(\left(B^{1}, B^{2}\right)\right)$ is equal to the image of $\left\{e_{1}, \ldots, e_{t-c}\right\}$ by the linear transformation ${ }^{t} S_{1}$ of Lemma 2.9 .

Lemma 2.11. The space $\Sigma$ is a differentiable submanifold of dimension $(t-c)(2 c-t)+2\left\{\frac{1}{2} c(c+1)+(t-c)(i-c)\right\}$ in the space of all pairs of $i$-th symmetric matrices:

Proof. The group $G L(c)$ operates $\Sigma$ naturally. Let $d=t-c$. Then we have the commutative diagram,

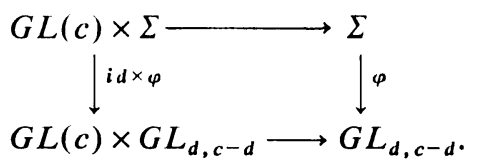

Hence $\varphi$ is a fibre bundle ([2, Appendix, Lemma 1]). Let $M$ be the space of matrices with the following form

$$
\left(\begin{array}{lll|l}
* & * & * & ) d \\
* & * & 0 & ) c-d \\
* & 0 & 0 & ) i-c
\end{array}\right.
$$


Then the fibre $F$ is an open manifold of $M^{2}$, since any element $\left(B^{1}, B^{2}\right)$ mapped on the space $\left\{e_{1}, \ldots, e_{t-c}\right\}$ in $G L_{t-c, 2 c-t}$ by $\varphi$ has the form given in Lemma 2.9 and ii), iii) and iv) are open conditions in $M^{2}$. Now we consider the differentiable map $\mu: G L(c) \times F \rightarrow A_{i} \times A_{i}$ where $A_{i}$ is the space of $i$-th symmetric matrices. By the similar argument as in the proof of Theorem 1, we can prove that $\mu$ is of constant rank and an open map and that the image of $\mu$ is $\Sigma$. Hence $\Sigma$ is a submanifold of dimension $\operatorname{dim} F+\operatorname{dim} G L_{t-c, 2 c-t}$. It is easy to see that $\operatorname{dim} F=c(c+1)+2(t-c)$ $(i-c)$.

Q.E.D.

\section{§3. Extensibility}

In [6] Andrew du Plessis introduced the notion of extensibility. This is defined as follows. Let $N$ and $P$ be differentiable manifolds. Let $\Omega$ be an open set of the jet space $\operatorname{Jr}(N, P)$. Then $\Omega$ is called extensible if there exists an open subbundle $\Omega^{\prime}$ of $J^{r}(N \times \mathbf{R}, P) \rightarrow N \times \mathbf{R}$, such that the following conditions hold for $\Omega^{\prime}$. i) A local diffeomorphism of $N \times \mathbf{R}, f: U \rightarrow V$ induces a diffeomorphism $j^{r} f$ : $J r(U, P) \rightarrow J^{r}(V, P)$ covering $f$. Then $\Omega^{\prime}$ is invariant by these induced diffeomorphisms $j^{r} f$.

ii) Let $i$ be the inclusion: $N=N \times 0 \rightarrow N \times \mathbf{R}$. Let $i^{*} J^{r}(N \times \mathbf{R}, P)$ denote the pull back of the canonical bundle: $\operatorname{Jr}^{r}(N \times \mathbf{R}, P) \rightarrow N \times \mathbf{R}$ by $i$. Then $i^{*} \Omega^{\prime}$ is carried onto $\Omega$ by the natural bundle map: $i^{*} J r(N \times \mathbf{R}, P) \rightarrow J r(N, P)$.

These conditions are a little stronger than those given in [6]. Condition i) is called naturality.

Theorem 3.1. (du Plessis [6]). If $\Omega$ is extensible, then

$$
j^{r}: C_{\Omega}^{\infty}(N, P) \longrightarrow \Gamma_{N}(\Omega)
$$

is a weak homotopy equivalence.

Remark 3.2. Since $\Sigma^{I}$ in $J^{r}(n, p)$ is invariant by the action of $L^{r}(n)$, so is $\Omega^{I}=\bigcup_{K \leqq I} \Sigma^{K}$. Hence $\Omega^{I}$ in $J^{r}(N, P)$ is a natural open set.

We will denote $\Sigma^{I}$ and $\Omega^{I}$ in $J^{r}(n, p)$ by $\Sigma^{I}(n, p)$ and $\Omega^{I}(n, p)$ in this section. We will determine the image of $i^{*} \Omega^{I}$ by the natural map: $i^{*} J^{2}(N \times \mathbf{R}, P) \rightarrow J^{2}(N$, $P)$ in terms of $\Sigma^{I}(t, c)$ in case of $p-n+i=2$. For this purpose it is enough to describe the image of $i^{*} \Omega^{I}(n+1, p)$. Let $M^{i, j+1}(n, p)$ in $J^{2}(n, p)$ be the set of $z$ such that $\mathrm{rk} \mathfrak{J}(z) \leqq n-i$ and $\mathrm{rk} \Delta^{n-i+1} \mathfrak{T}(z) \leqq n-j-1(n-i \leqq n-j-1)$. Note that $\Delta^{n-i+1} \mathfrak{I}(z)$ is not necessarily $\delta \mathfrak{I}(z)$ in this case. Let $I$ be $(i, j)$. Then $\Omega^{I}(n, p)$ $=\bigcup_{K \leqq I} \Sigma^{K}(n, p)$.

Lemma 3.3. $J^{2}(n, p)-M^{i, j+1}(n, p)=\Omega^{I}(n, p)$.

Proof. We will prove the equivalent assertion that $M^{i, j+1}(n, p)=\bigcup_{K>I} \Sigma^{K}$. Let $z$ be in the union of $\Sigma^{K}, K>I$. If $\mathrm{rk} \mathfrak{\Im}(z)<n-i$, then $\Delta^{n-i+1} \mathfrak{T}(z)=\mathfrak{\Im}(z)$ modulo 
$\mathfrak{M}^{2}$. Hence rk $\Delta^{n-i+1} \mathfrak{I}(z)<n-i \leqq n-j-1$. If $\mathrm{rk} \mathfrak{I}(z)=n-i$, then $\Delta^{n-i+1} \mathfrak{I}(z)$ $=\delta \mathfrak{I}(z)$ by definition. Hence it follows from $\mathrm{rk} \delta \mathfrak{I}(z) \leqq n-j-1$ that $z \in M^{i, j+1}(n$, $p)$. Conversely for any element $z$ of $M^{i, j+1}(n, p)$ we have $\operatorname{rk} \mathfrak{I}(z) \leqq n-i$ and rk $\Delta^{n-i+1} \mathfrak{T}(z) \leqq n-j-1$. If rk $\mathfrak{T}(z)<n-i$, then $z \in \cup_{K>I} \Sigma^{K}$. If $\mathrm{rk} \mathfrak{T}(z)=n-i$, and rk $\Delta^{n-i+1} \mathfrak{I}(z)=\mathrm{rk} \delta \mathfrak{I}(z) \leqq n-j-1$, then $z$ is contained in $\underset{K>I}{\cup} \Sigma^{K}$. $\quad$ Q. E. D.

Let $\tilde{z}$ be an element of $J^{2}(n+1, p)$ and its representative $\tilde{f}: \mathbf{R}^{n+1} \rightarrow \mathbf{R}^{p}$. Then we let $f(x)=f(x, 0), x \in \mathbf{R}^{n}$ and $z$, the element of $J^{2}(n, p)$ represented by $f$. A natural map $i: J^{2}(n+1, p) \rightarrow J^{2}(n, p)$ is defined by $i(\tilde{z})=z$. By definition it is easy to see that $M^{i, j+1}(n+1, p)$ is an algebraic set of $J^{2}(n+1, p)$. Hence $J^{2}(n, p)$ $-i\left(\Omega^{I}(n+1, p)\right)$ is closed in Zariski topology, so it is a finite union of differentiable manifolds ([9]). In this section we show that $J^{2}(n, p)-i\left(\Omega^{I}(n+1, p)\right)$ is explicitly described in terms of $\Sigma^{I}(t, c)$ when $p-n+i=2$.

Lemma 3.4. Let $p-n+i>1$. Then $z$ is an element of $J^{2}(n, p)-i\left(\Omega^{i, j}(n+1\right.$, $p)$ ) if and only if rk $\mathfrak{T}(z) \leq n-i$ and $\mathrm{rk} \Delta_{a}^{n-i+2} \mathfrak{I}(z) \leq n-j-1$ for any $a \in \mathbf{R}^{p}$.

Proof. By the definition of $\Delta_{a}^{n-i+2}$, the last statement is equivalent to say that i) $\mathrm{rk} \mathfrak{I}(z)<n-i$ or ii) $\mathrm{rk} \mathfrak{I}(z)=n-i$ and $\operatorname{rk} \delta_{a} \mathfrak{I}(z) \leq n-j-1$ for any $a \in \mathbf{R}^{p}$. Note that $z$ is an element of $J^{2}(n, p)-i\left(\Omega^{I}(n+1, p)\right)$ if and only if for any $\tilde{z}$ with $i(\tilde{z})=z, \tilde{z}$ is contained in $M^{i, j+1}(n+1, p)$. By Lemma 3.3 the last statement is equivalent to say that for any $\tilde{z}$ with $i(\tilde{z})=z$, i) $\operatorname{rk} \mathfrak{I}(\tilde{z})<n-i+1$ or ii) $\operatorname{rk} \mathfrak{I}(\tilde{z})=n-i+1$ and $\operatorname{rk} \delta \Im(\tilde{z}) \leqq n-j$. Let $f$ and $\tilde{f}$ be representatives of $z$ and $\tilde{z}$. Let $f$ be written in the form of (2.6) and $\tilde{f}$, as follows,

$$
f_{i}\left(x, x_{n+1}\right)=\sum_{i=1}^{n} a_{j}^{i} x_{j}+a^{i} x_{n+1}+\sum_{s, t \leqq n+1} b_{s t}^{i} x_{s} x_{t} \quad(i=1, \ldots, p) .
$$

Then the rank of the Jacobian matrix $\left(\partial f_{i} / \partial x_{j}\right)$ is equal to rk $D(f, x, a)$ at $x=0$. Let $k<\min (n, p)$. Then $\operatorname{rk} \Im(z)=k$ if and only if $\operatorname{rk} D(f, x, a) \leqq k+1$ and $\operatorname{rk} D(f, x, b)$ $=k+1$ for some $b$ at $x=0$. Hence if $p-n+1>1$, then $i) \mathrm{rk} \Im(z)<n-i$ if and only if rk $\mathfrak{I}(\tilde{z})<n-i+1$ for any $\tilde{z}$ with $i(\tilde{z})=z$ and ii) $\mathrm{rk} \mathfrak{I}(z)=n-i$ if and only if rk $\mathfrak{I}(\tilde{z})$ $\leqq n-i+1$ for any $z$ with $i(\tilde{z})=z$ and $\operatorname{rk} \Im\left(\tilde{z}_{0}\right)=n-i+1$ for some $\tilde{z}_{0}$. Hence it is enough to prove the case of $\operatorname{rk} \mathfrak{I}(z)=n-i$. If $\operatorname{rk} \mathfrak{I}(\tilde{z})=n-i+1$, then $\delta \mathfrak{I}(\tilde{z})$ is generated by $\delta_{a} \mathfrak{I}(z)$ and $x_{n+1}$ modulo $\mathfrak{M}^{2}$. Hence $\operatorname{rk} \delta \mathfrak{I}(z)=\operatorname{rk} \delta_{a} \mathfrak{I}(z)+1$. Q. E. D.

Proposition 3.5. Let $p-n-i=2$. Then $i\left(\Omega^{i, j}(n+1, p)\right)$ is $\Omega^{i, j}(n, p)-$ $\left(\underset{\substack{h \leqq j \\ j-i+i<0 \\ c \leqq t}}{\bigcup} \Sigma^{i, h}(t, c)\right)$.

Proof. By Lemma $3.4 i\left(\Omega^{I}(n+1, p)\right)$ is the set of all elements $z$ such that i) rk $\mathfrak{I}(z)>n-i$ or ii) $\operatorname{rk} \mathfrak{I}(z)=n-i$ and $\Delta_{a}^{n-i+2} \mathfrak{I}(z) \geqq n-j$ for some $a \in \mathbf{R}^{p}$. The maximal rank of $\Delta_{a}^{n-i+1} \mathfrak{T}(z), a \in \mathbf{R}^{p}$ is $n-i+t$ for an element $z \in \Sigma^{i, h}(t, c)$. Hence $i\left(\Omega^{I}(n+1, p)\right)=\bigcup_{k<i} \Sigma^{k, h}(n, p) \cup\left(\underset{\substack{h \leqq j \\ j-i+t \geq 0 \\ c \geqq t}}{\bigcup} \Sigma^{i, h}(t, c)\right)$.

Since $t \leqq i-h$, we have $j \geqq h$. Hence 


$$
i\left(\Omega^{I}(n+1, p)\right)=\Omega^{I}(n, p)-\left(\underset{\substack{h \leqq j \\ j-i+t<0 \\ c \leqq t}}{\bigcup} \sum^{i, h}(t, c)\right) .
$$

Q.E.D.

Proof of $(v)$ of Theorem. Note that $\Sigma^{I}(t, c)=\bigcup_{x \in N, y \in P} \Sigma_{y}^{I}(t, c)_{x, y}$. It follows from Proposition 3.5 that $i\left(\Omega^{I}(N \times R, P)\right)$ is extensible. Hence $(v)$ of Theorem follows from Theorem 3.1.

Q.E.D.

\section{§4. Proof of Corollary}

Let $\Omega(N)$ (or $\Omega^{\prime}(N)$ ) be an open subbundle of $J^{r}(N, P) \rightarrow N$ such that $\Omega(N)$ $-\Omega^{\prime}(N)$ (or $\Omega^{\prime}(N)-\Omega(N)$ ) is a differentiable subbundle whose fiber over $(x, y)$ is a finite union of submanifolds of $J^{r}(N, P)_{x, y}$ with codimensions in $J^{r}(N, P)_{x, y}$ greater than $n+\sigma_{1}$ (or $n+\sigma_{2}$ ). In [5] A. du Plessis has shown the following lemma.

Transversality Lemma ([5]). Let $\Omega(N)$ and $\Omega^{\prime}(N)$ be as above. Let $\Omega^{\prime}(N)$ be integrable. (i) Let $\sigma_{1} \leqq \sigma_{2}-1$. Then $\left(j^{r}\right)_{*}: \pi_{i}\left(C_{\Omega(N)}^{\infty}(N, P)\right) \rightarrow \pi_{i}\left(\Gamma_{N}(\Omega(N))\right)$ is isomorphic for $i<\sigma_{1}$ and surjective for $i=\sigma_{1}$. (ii) Let $\sigma_{2}-1<\sigma_{1}$. Then $\left(j^{r}\right)_{*}$ : $\pi_{i}\left(C_{\Omega(N)}^{\infty}(N, P)\right) \rightarrow \pi_{i}\left(\Gamma_{N}(\Omega(N))\right)$ is isomorphic for $i<\sigma_{2}$.

We give here two consequences of Transversality lemma.

Theorem 4.1. Let $\Omega^{I}$ be an integrable subset of $J^{r}(N, P)$. i) Let $K \geqq I$. We define $\sigma_{1}$ to be $\sigma_{1}+n+1=\min \left\{\operatorname{codim} \Sigma^{H}\right.$ in $\left.\operatorname{Jr}(N, P) \mid K \geqq H>I\right\}$. Then $\left(j^{r}\right)_{*}$ : $\pi_{i}\left(C_{\Omega^{K}}^{\infty}(N, P)\right) \rightarrow \pi_{i}\left(\Gamma_{N}\left(\Omega^{K}\right)\right)$ is isomorphic for $i<\sigma_{1}$ and surjective for $i=\sigma_{1}$.

ii) Let $K \leqq I$. We define $\sigma_{2}$ to be $\sigma_{2}+n+1=\min \left\{\operatorname{codim} \Sigma^{H}\right.$ in $\left.J^{r}(N, P) \mid I \geqq H>K\right\}$. Then $\left(j^{r}\right)_{*}: \pi_{i}\left(C_{\Omega^{K}}^{\infty}(N, P)\right) \rightarrow \pi_{i}\left(\Gamma_{N}\left(\Omega^{K}\right)\right)$ is isomorphic for $i<\sigma_{2}$.

Proof. We only prove the first part of the theorem since the proof of the second part is similar. In (i) of Transversality lemma we consider $\Omega^{K}$ and $\Omega^{I}$ instead of $\Omega(N)$ and $\Omega^{\prime}(N)$. Then $\Omega^{K}-\Omega^{I}$ is the union of $\Sigma^{H}, I<H \leqq K$ with codimensions greater than $\sigma_{1}+n$.

Q.E.D.

We should note that $\sigma_{2}$ is greater than $\sigma_{1}$ in most cases.

The following corollary follows from Theorem in $\S 0$ and includes Corollary in $\S 0$ since $\operatorname{codim} \Sigma^{i, 1}=3 i+1$.

Corollary 4.2. Let $r=2$ and $I=(i, j)$. If $p-n+i=2$, then $\left(j^{2}\right)_{*}: \pi_{k}\left(C_{\Omega^{J}}^{\infty}(N\right.$, $P)) \rightarrow \pi_{k}\left(\Gamma_{N}\left(\Omega^{I}\right)\right)$ is isomorphic for $k<\operatorname{codim} \Sigma^{i, j+1}-j-n-1$ and surjective for $k=\operatorname{codim} \Sigma^{i, j+1}-j-n-1$.

Proof. In (i) of Transversality lemma we consider $\Omega^{I}$ and $\Omega^{I}-\underbrace{}_{\substack{h \leqq j \\ j-i+j \\ c \leqq t \\ c \leq t}} \Sigma^{i, h}(t, c)$ instead of $\Omega(N)$ and $\Omega^{\prime}(N)$. Then $\Omega(N)-\Omega^{\prime}(N)$ is the union of $\Sigma^{i, h}(t, c)$ with $h \leqq j, j-i+t<0$ and $c \leqq t$. It is easy to see that $\operatorname{codim} \Sigma^{i, h}(t, c) \geqq \operatorname{codim} \Sigma^{i, j+1}-j$.

Q.E.D.

Examples. i) $J^{2}(3,2) ; \Omega^{I}$ for $I=(3,0),\left(2, j_{2}\right)$ and $(1,1)\left(j_{2}<2\right)$ are not 
extensible. $\quad \Sigma^{3,0}=\Sigma^{3,0}(3,3) \cup \Sigma^{3,0}(2,1)$.

ii) $J^{2}(4,2) ; \Omega^{I}$ for $I=\left(4, j_{1}\right),\left(3, j_{2}\right)$ and $(2,2)\left(j_{1} \leqq 1, j_{2}<3\right)$ are not extensible. $\Sigma^{4,0}=\Sigma^{4,0}(4,4) \cup \Sigma^{4,0}(3,2) . \quad \Sigma^{4,1}=\Sigma^{4,1}(3,3) \cup \Sigma^{4,1}(2,1)$.

iii) $J^{2}(5,2) ; \Omega^{I}$ for $I=\left(5, j_{1}\right),\left(4, j_{2}\right)$ and $(3,3)\left(j_{1} \leqq 2, j_{2}<4\right)$ are not extensible. $\Sigma^{5,2}=\Sigma^{5,2}(3,3) \cup \Sigma^{5,2}(2,1) . \quad \Sigma^{5,1}=\Sigma^{5,1}(4,4) \cup \Sigma^{5,1}(3,2) . \quad \Sigma^{5,0}=\Sigma^{5,0}(5,5) \cup \Sigma^{5,0}$ $(4,3) \cup \Sigma^{5,0}(4,2)$.

iv) $J^{2}(6,2) ; \Omega^{I}$ for $I=\left(6, j_{1}\right),\left(5, j_{2}\right)$ and $(4,4)\left(j_{1} \leqq 3, j_{2}<5\right)$ are not extensible. $\Sigma^{6,3}=\Sigma^{6,3}(3,3) \cup \Sigma^{6,3}(2,1) . \quad \Sigma^{6,2}=\Sigma^{6,2}(4,4) \cup \Sigma^{6,2}(3,2) . \quad \Sigma^{6,1}=\Sigma^{6,1}(5,5) \cup \Sigma^{6,1}$ $(4,3) \cup \Sigma^{6,1}(4,2) . \quad \Sigma^{6,0}=\Sigma^{6,0}(6,6) \cup \Sigma^{6,0}(5,4) \cup \Sigma^{6,0}(5,3) \cup \Sigma^{6,0}(4,2)$.

We give a table of $\sigma_{1}, \sigma_{2}$ and $\xi=\operatorname{codim} \Sigma^{i, j+1}-j-n-1$ for some cases (In the table $(i,-1)$ means $(i-1, i-1))$.

\begin{tabular}{c|c|rr|rrr|rrrr}
$(n, p)$ & $J^{2}(3,2)$ & \multicolumn{2}{|c|}{$J^{2}(4,2)$} & \multicolumn{3}{|c|}{$J^{2}(5,2)$} & \multicolumn{4}{c}{$J^{2}(6,2)$} \\
\hline$I$ & 3,0 & 4,1 & 4,0 & 5,2 & 5,1 & 5,0 & 6,3 & 6,2 & 6,1 & 6,0 \\
\hline$\xi$ & 6 & 8 & 8 & 20 & 15 & 10 & 30 & 24 & 17 & 12 \\
$\sigma_{1}$ & 4 & 8 & 8 & 4 & 4 & 4 & 5 & 5 & 5 & 5 \\
$\sigma_{2}$ & 6 & 13 & 8 & 26 & 16 & 10 & 33 & 26 & 19 & 12 \\
\hline$I$ & $2, j$ & \multicolumn{1}{|c|}{$3, j$} & \multicolumn{1}{c|}{$4, j$} & \multicolumn{4}{|c|}{$5, j$} \\
\hline$\sigma_{2}$ & $3 j+3$ & $4 j+5$ & \multicolumn{3}{|c|}{$5 j+7$} & \multicolumn{4}{|c|}{$6 j+9$}
\end{tabular}

Department of Mathematics HOKKAIDO UNIVERSITY

\section{References}

[1] J. M. Boardman, Singularities of differentiable maps, Publ. I. H. E. S., No. 33 (1967), 21-57.

[ 2] J. Cerf, Sur les difféomorphismes de la sphère de dimension trois $\left(\Gamma_{\mathbb{a}}=0\right)$, Springer Lecture Note, 53.

[ 3 ] J. Dieudonné, Foundations of modern Analysis, Academic Press, New York, 1960.

[4] A. A. du Plessis, Maps without certain singularities, Comment. Math. Helv., 50 (1975), 363-382.

[5] A. A. du Plessis, Contact invariant regularity conditions, Springer Lecture Note 535, 205-236.

[6] A. A. du Plessis, Homotopy classification of regular sections, preprint.

[7] J. N. Mather, On Thom-Boardman singularities, Dynamical Systems, Academic Press, 1973, 233-248.

[8] R. Thom, Les singularités des applications différentiables, Ann. Inst. Fourier, 6 (1956), 43-87.

[9] H. Whitney, Elementary structure of real algebraic varieties, Ann. of Math., 66 (1956), 545-556. 